\title{
Dinâmica populacional de Triportheus albus, T. angulatus e T. auritus (CHARACIFORMES: CHARACIDAE) em lagos da Amazônia Central
}

\author{
Luiza Prestes ${ }^{1,3}$, Maria Gercilia Mota Soares ${ }^{1}$, Fabio Ribeiro Silva ${ }^{2} \&$ Maria Mercedes Bittencourt ${ }^{1}$ \\ ${ }^{1}$ Coordenação de Pesquisas em Biologia Aquática, Instituto Nacional de Pesquisas da Amazônia - INPA, \\ Av. Andre Araujo, 2936-Aleixo, CEP 69060-001, Manaus, AM, Brasil \\ ${ }^{2}$ Museu Paraense Emilio Goeldi - MPEG (Zoologia), \\ Av. Perimetral, 1901-Terra Firme, CEP 66077-830, Belém, PA, Brasil \\ ${ }^{3}$ Autora para correspondência: Luiza Prestes, e-mail: luliprestes@gmail.com
}

PRESTES, L., SOARES, M.G.M., SILVA, F.R. \& BITTENCOURT, M.M. Dynamic population from Triportheus albus, T. angulatus and T. auritus (CHARACIFORMES: CHARACIDAE) in Amazonian Central lakes. Biota Neotrop. 10(3): http://www.biotaneotropica.org.br/v10n3/en/abstract?article+bn03210032010.

\begin{abstract}
In the Amazon the fishing is considered an important source of income and animal protein for the people in the region. Among the different fish species that landed in the market and fairs stand out Triportheus albus, T. angulatus and T. auritus. Considering the reduction of some fish stocks in the region, both the quantity and the size of some species, the research proposes to determine the growth parameters, mortality and length-weight relation of these species in floodplain lakes, Manacapuru, AM. The parameters were estimated by analyzing the length frequency distribution with the help of the routine "scan of values of k" method ELEFAN I program FISAT II. The values for each species were: . albus $\mathrm{L}_{\infty}=24.68 \mathrm{~cm}, \mathrm{k}=0.86 /$ year, $\varnothing^{\circ}=2.71, \mathrm{~A}_{0.95}=3.48$ years, $\mathrm{Mp}=1.76 /$ year, $\mathrm{Mt}=0.85 /$ year, $\mathrm{Pt}=0.0398 * \mathrm{Cp}^{2.6303} ;$ T. angulatus $\mathrm{L}_{\infty}=26.78 \mathrm{~cm}, \mathrm{k}=0.77 /$ year, $\varnothing{ }^{\prime}=2.74$, $\mathrm{A}_{0.95}=3.89$ years, $\mathrm{Mp}=1.60 /$ year, $\mathrm{Mt}=0.76 /$ year, $\mathrm{Pt}=0.0294 * \mathrm{Cp}^{2.8599}$ and T. auritus $\mathrm{L}_{\infty}=27.83 \mathrm{~cm}, \mathrm{k}=0.65 /$ year, $\varnothing:=2.70, \mathrm{~A}_{0.95}=4.68$ years, $\mathrm{Mp}=1.40 /$ year, $\mathrm{Mt}=0.63 /$ year and $\mathrm{Pt}=0.0542 * \mathrm{Cp}^{2.5094}$. These species showed rapid growth, high mortality, low life expectancy and short life cycle. It is suggested the parameters population should be continuously monitored to be used as a tool in the stock analysis.

Keywords: growth, mortality, weight-length relationship, freshwater fishes.
\end{abstract}

PRESTES, L., SOARES, M.G.M., SILVA, F.R. \& BITTENCOURT, M.M. Dinâmica populacional de Triportheus albus, T. angulatus e T. auritus (CHARACIFORMES: CHARACIDAE) em lagos da Amazônia Central. Biota Neotrop. 10(3): http://www.biotaneotropica.org.br/v10n3/pt/abstract?article+bn03210032010.

Resumo: Na Amazônia brasileira a pesca é importante fonte de renda e proteína animal para as populações ribeirinhas. E, entre as espécies de peixes de maior volume desembarcado nos mercados e feiras da região se destacam as sardinhas, Triportheus albus, T. angulatus e T. auritus. Considerando o aumento da pressão pesqueira, que gera a redução de alguns estoques pesqueiros na região, tanto na quantidade como no tamanho de algumas espécies, o trabalho propõe determinar os parâmetros de crescimento, mortalidade e relação pesocomprimento dessas espécies em lagos de várzea, Manacapuru, AM. Os parâmetros foram estimados através da análise de distribuição de frequência de comprimento com o auxílio da rotina "scan de valores de k" do método ELEFAN I do programa FISAT II. Os valores calculados foram: . albus $\mathrm{L}_{\infty}=24,68 \mathrm{~cm}, \mathrm{k}=0,86 /$ ano, $\varnothing^{\prime}=2,71$, $\mathrm{A}_{0,95}=3,48$ anos, $\mathrm{Mp}=1,76 / \mathrm{ano}, \mathrm{Mt}=0,85 / \mathrm{ano}, \mathrm{Pt}=0,0398 * \mathrm{Cp}^{2,6303} ;$ T. angulatus $\mathrm{L}_{\infty}=26,78 \mathrm{~cm}, \mathrm{k}=0,77 /$ ano, $\varnothing^{\prime}=2,74, \mathrm{~A}_{0,95}=3,89$ anos, $\mathrm{Mp}=1,60 / \mathrm{ano}, \mathrm{Mt}=0,76 /$ ano, $\mathrm{Pt}=0,0294 * \mathrm{Cp}^{2,8599} ;$ T. auritus $\mathrm{L}_{\infty}=27,83 \mathrm{~cm}$, $\mathrm{k}=0,65 / \mathrm{ano}, \varnothing^{\prime}=2,70, \mathrm{~A}_{0,95}=4,68$ anos $\mathrm{Mp}=1,40 / \mathrm{ano}, \mathrm{Mt}=0,63 /$ ano e $\mathrm{Pt}=0,0542 * \mathrm{Cp}^{2,5094}$. Essas espécies apresentaram crescimento rápido, mortalidade elevada, baixa expectativa de vida e ciclo de vida curto. É sugerido o monitoramento desses parâmetros para que possam ser utilizados como instrumentos de análise dos estoques pesqueiros.

Palavras-chave: crescimento, mortalidade, relação peso-comprimento, peixes de água doce. 


\section{Introdução}

Na Amazônia brasileira a pesca é uma das principais atividades extrativistas, que abastece os mercados de vários estados do Brasil e outros países (Petrere Jr. 1992, Ibama 2008), e isso suporta o consumo de pescado, considerado maior do que em outras regiões (Smith 1979, Batista 1998). A atividade pesqueira explora cerca de 200 espécies de peixes (Barthem \& Petrere Jr. 1995), mas a pesca comercial está concentrada em 31 categorias de peixes, que são responsáveis pelos maiores volumes de pescado comercializado na região (Batista \& Petrere Jr. 2003, Ruffino et al. 2006). Porém, o conhecimento sobre a dinâmica das populações destas espécies ainda é limitado, especialmente considerando a alta riqueza de peixes e os altos níveis de produção biológica que caracteriza as áreas alagáveis dos rios de águas brancas.

Na Amazônia Central estudos sobre dinâmica abordando o crescimento foram realizados determinando a idade dos peixes por meio de leitura de estruturas calcificadas em matrinxã, Brycon amazonicus (Spix \& Agassiz, 1829) (Werder 1983), aracu, Schizodon fasciatus Spix \& Agassiz, 1829 (Fabré \& Saint-Paul 1998), tambaqui, Colossoma macropomum (Cuvier, 1816) (Penna et al. 2005), mapará, Hypophthalmus marginatus Valenciennes, 1840 (Cutrim \& Batista 2005) e piracatinga, Calophysus macropterus (Lichtenstein, 1819) (Perez \& Fabré 2009), a maioria de estoques pesqueiros de importância comercial. E, por causa da dificuldade de se trabalhar com estas estruturas, desde a década de 80 , métodos indiretos baseados na frequência de comprimento vêm sendo utilizados para a obtenção de informações da dinâmica de estoques pesqueiros. Alguns softwares têm facilitado a análise dos dados, como o FISAT. Os métodos indiretos já foram utilizados nas estimativas de parâmetros populacionais de piranha-caju, Pygocentrus nattereri Kner, 1858 (Pauly 1994, Soares et al. 2009), tambaqui, C. macropomum (Petrere Jr. 1983, Isaac \& Ruffino 1996, Soares et al. 2009), curimatã, Prochilodus nigricans Spix \& Agassiz, 1829, aracu, S. fasciatus, surubim-lenha, Pseudoplatystoma tigrinum (Valenciennes, 1840) surubim-tigre, Pseudoplatystoma punctifer (Linnaeus, 1766), dourada, Brachyplatystoma flavicans (Castelnau, 1855), mapará, H. marginatus (Ruffino \& Isaac 1995) e tucunaré Cichla monoculus Spix \& Agassiz, 1831 (Ruffino \& Isaac 1995, Soares et al. 2009).

Nos portos de desembarque de pescado no Amazonas, espécies de peixes que em anos anteriores faziam ocasionalmente parte da comercialização, hoje têm maior participação. É o caso das sardinhas, Triportheus albus (Cope 1872), Triportheus angulatus (Spix \& Agassiz 1829) e Triportheus auritus (Valenciennes 1850), que na década de 70 eram pouco comercializadas, 2\% (Petrere Jr. 1978, Merona \& Bittencourt 1988), e atualmente estão entre os peixes de maior volume, $12 \%$, desembarcado no porto de Manaus e de Manacapuru, AM (Ruffino et al. 2006, Gonçalves \& Batista 2008).

Considerando o atual aumento da pressão pesqueira, que gera a redução de alguns estoques pesqueiros na região, tanto na quantidade como no tamanho de algumas espécies, o presente estudo propõe determinar os parâmetros de crescimento e mortalidade de T. albus, T. angulatus e T. auritus capturadas em lagos de várzea da Amazônia Central. Estas informações deverão ser utilizadas como ferramentas na tomada de decisões sobre a gestão das unidades populacionais desses peixes no Complexo lacustre lago Grande de Manacapuru, AM.

\section{Materiais e Métodos}

Os peixes foram capturados mensalmente de julho de 2006 a abril de 2008 nos lagos Jaitêua (03 13' 901' S e 60 44'326” W) e São Lourenço $\left(03^{\circ} 17^{\prime} 555^{\prime \prime}\right.$ S e $60^{\circ} 43$ ' 759' W), localizados no Complexo lacustre lago Grande de Manacapuru, AM (Figura 1). As amostragens foram efetuadas na região de água aberta e de floresta alagada, com baterias de malhadeiras, tamanhos de malha variando

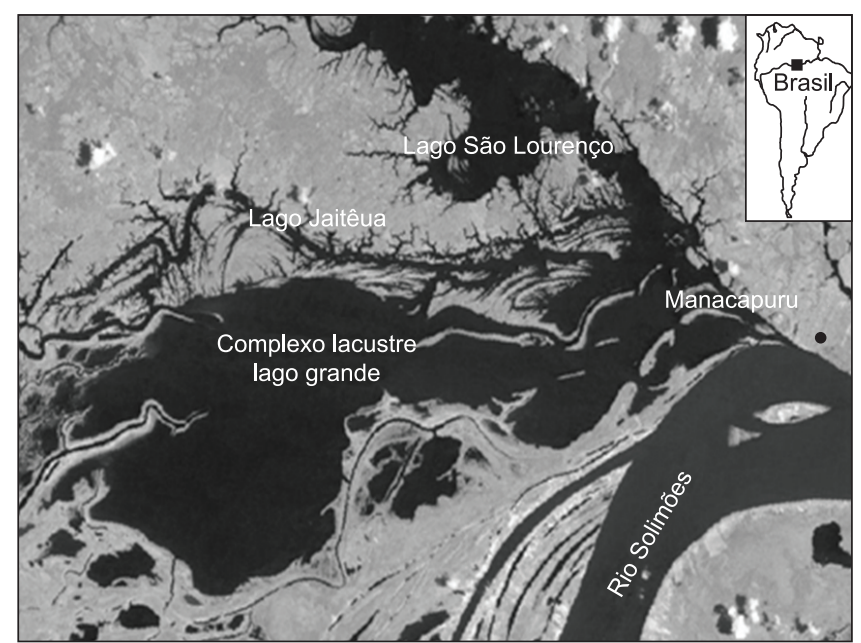

Figura 1. Localização dos lagos Jaitêua e São Lourenço, integrantes do Complexo lacustre Lago grande, Rio Solimões, AM.

Figure 1. Location of Jaitêua and São Lourenço lakes at the lago Grande de Manacapuru lacustrine complex, Solimões River, AM.

de 20 a $120 \mathrm{~mm}$ entre nós opostos, expostas durante 24 horas com despescas a cada 6 horas. Os exemplares capturados foram identificados no campo, registrados as datas de coleta, comprimento padrão em cm $\left(\mathrm{C}_{\mathrm{p}}\right)$ e peso total em gr $\left(\mathrm{P}_{\mathrm{t}}\right)$.

\section{Análise dos Dados}

O crescimento foi estimado com base nas distribuições de frequência mensais de comprimento (classes de $1 \mathrm{~cm}$ ). Os parâmetros de crescimento da equação de von Bertalanffy $\mathrm{L}=\mathrm{L}_{\infty} *\left[1-e^{(-k(t-t))}\right]$, $\mathrm{L}_{\infty}=$ comprimento assintótico e $\mathrm{k}=$ taxa de crescimento foram estimados através da rotina ELEFAN I do Programa FAO-ICLARM Stock Assessment Tools - FISAT (Gayanilo et al. 1996). A partir da rotina "scan de valores de k" foram realizados vários ensaios para obter melhores ajustes das curvas de crescimento, aliando os valores resultantes de $\mathrm{k}, \mathrm{L}_{\infty}$ e o número de coortes que passam pelos picos modais (Angelini \& Gomes 2008). Nestas análises $\mathrm{t}_{0}$ foi considerado zero, pois não é um parâmetro biológico, mas, um mecanismo matemático para tornar a curva de crescimento melhor ajustada (Moreau 1987).

$\mathrm{O}$ índice de performance de crescimento $\left(\varnothing^{\prime}\right)$ foi estimado por Pauly e Munro (1984): $\varnothing^{\prime}=\log (\mathrm{k})+2 * \log \left(\mathrm{L}_{\infty}\right)$, a longevidade $\left(\mathrm{A}_{0,95}\right)$ por Taylor (1958): $\mathrm{A}_{0,95}=\mathrm{t}_{0}+(2,996 / \mathrm{k})$ e a mortalidade natural (M) foi estimada pelo método de Taylor (1958) $\mathrm{MT}=-\ln (1-0,95) / \mathrm{A}_{0.95}$ e pela fórmula empírica de Pauly (1980) $\operatorname{lnMP}=-0,0152-0,279 * \ln \mathrm{L}_{\infty}+0,6543 * \operatorname{lnk}+0,463 * \ln \mathrm{T}$, onde $\mathrm{T}$ é temperatura média da superfície, nesse caso $30^{\circ} \mathrm{C}$.

A relação peso-comprimento foi estimada pela expressão $\mathrm{Pt}=\mathrm{a}^{*} \mathrm{Cp}^{\mathrm{b}}$, após transformação logarítmica, sendo: Pt o peso total, Cp o comprimento padrão, a o intercepto da curva e b o coeficiente de alometria da relação peso-comprimento. O tipo de crescimento foi verificado através do teste- $t$ onde: $\mathrm{H}_{0}: \mathrm{b}=3$ (crescimento isométrico) e $\mathrm{H}_{1}: \mathrm{b} \neq 3$ (crescimento alométrico) $(\alpha=0.05)$ (Zar 1996).

\section{Resultados}

Nos lagos foram capturados 693 exemplares de T. albus, 665 de T. angulatus e 486 de T. auritus com comprimento padrão variando entre 8,5 e $23,5 \mathrm{~cm}, 7,0$ e $25,5 \mathrm{~cm}$ e 6,5 e $26,5 \mathrm{~cm}$, respectivamente. A dispersão dos dados para cada espécie apresentou uma curva normal com médias e desvio padrão de 14,8 $\pm 1,93,14,9 \pm 2,80$ e $16,3 \pm 3,23$, respectivamente. 
O melhor ajuste dos parâmetros de crescimento, estimativas de mortalidade e da relação peso-comprimento para as espécies foi: T. albus $\mathrm{L}_{\infty}=24,68 \mathrm{~cm}, \mathrm{k}=0,86 / \mathrm{ano}, \varnothing^{\prime}=2,71, \mathrm{~A}_{0,95}=3,48$ anos, $\mathrm{Mp}=1,76 / \mathrm{ano}, \mathrm{Mt}=0,85 / \mathrm{ano}, \mathrm{Pt}=0,0398 * \mathrm{Cp}^{2,6303} ;$ T. angulatus $\mathrm{L}_{\infty}=26,78 \mathrm{~cm}, \mathrm{k}=0,77 / \mathrm{ano}, \varnothing^{\prime}=2,74, \mathrm{~A}_{0.95}=3,89$ anos, $\mathrm{Mp}=1,60 /$ ano, $\mathrm{Mt}=0,76 / \mathrm{ano}, \mathrm{Pt}=0,0294 * \mathrm{Cp}^{2,8599}$ e $T$. auritus $\mathrm{L}_{\infty}=27,83 \mathrm{~cm}, \mathrm{k}=0,65 / \mathrm{ano}, \varnothing^{\prime}=2,70, \mathrm{~A}_{0,95}=4,68$ anos, $\mathrm{Mp}=1,40 / \mathrm{ano}$, $\mathrm{Mt}=0,63 /$ ano, $\mathrm{Pt}=0,0542 * \mathrm{Cp}^{2,5094}$ (Tabela 1, Figura 2).

A relação peso-comprimento calculada apontou crescimento alométrico negativo, $\mathrm{b}<3$, para T. albus $\left(\mathrm{t}^{<} \mathrm{t}_{2(0.05) 683} ; \mathrm{p}<0,05\right)$, T. angulatus $\left(\mathrm{t}^{<} \mathrm{t}_{2(0,05) 629} ; p<0,05\right)$ e T. auritus $\left(\mathrm{t}<\mathrm{t}_{2(0,05) 472} ; \mathrm{p}<0,05\right)$ (Tabela 1, Figura 2).

\section{Discussão}

Na Amazônia, são escassas informações sobre dinâmica populacional das espécies de peixe. Especificamente, para T. albus, T. angulatus e T. auritus, até o presente momento na literatura especializada não há informações sobre crescimento, mortalidade e relação peso-comprimento. Assim, estes são os primeiros valores estimados para essas espécies.

Os exemplares de T. albus $(8,5 \mathrm{a} 23,5 \mathrm{~cm}), T$. angulatus $(7 \mathrm{a} 25,5 \mathrm{~cm})$ e T. auritus $(6,5$ a $26,5 \mathrm{~cm}$ ) capturados no Jaitêua e São Lourenço foram relativamente maiores do que aqueles capturados nos lagos Castanho (Almeida 1984), Camaleão (Yamamoto et al. 2004) e Rei (Rubiano 1999) (Tabela 2). Esse resultado pode ser explicado pelo fato das pescarias no Jaitêua e São Lourenço terem sido realizadas com tamanho de malha (20 a $120 \mathrm{~mm}$ ) maior do que as utilizadas nos outros lagos. Também, deve ser levado em consideração que o Jaitêua e São Lourenço fazem parte de um complexo lacustre constituído de lagos, paranás, igarapés e furos. Essa condição favorece a existência de muitas áreas de alimentação, abrigo e reprodução para os peixes (Soares et al. 2009).
Tabela 1. Número de peixes utilizados na análise $(\mathrm{N})$, intervalo de comprimento $($ Lmáx $=$ maior comprimento capturado; Lmín $=$ menor comprimento capturado), parâmetros de crescimento ( $\mathrm{k}=$ taxa de crescimento; $\mathrm{L}_{\infty}=$ comprimento assintótico; $\varnothing^{\prime}=$ índice de performance de crescimento), longevidade $\left(\mathrm{A}_{0.95}=\right.$ idade máxima $)$ mortalidade $(\mathrm{Mp}=$ Mortalidade por Pauly; $\mathrm{Mt}=$ Mortalidade por Taylor $)$ e relação peso-comprimento $(\mathrm{a}=$ coeficiente linear; $\mathrm{b}=$ coeficiente de alometria) de T. albus, T. angulatus e T. auritus.

Table 1. Number of fishes $(\mathrm{N})$, interval lenght (Lmax. = bigger lenght caught; Lmin. = lesser lenght caught), growth parameters $(\mathrm{k}=$ coefficient growth; $\mathrm{L}_{\infty}=$ asymptotic lenght; $\varnothing^{\prime}=$ growth performance index $)$, longevity $\left(\mathrm{A}_{0.5}=\right.$ maximal age $)$, mortality $(\mathrm{Mp}=$ Mortality Pauly method; $\mathrm{M}=$ Mortality Taylor method) and lenght-weight relation ( $\mathrm{a}=$ intercept; $\mathrm{b}=$ slope $)$ of T. albus, T. angulatus and T. auritus.

\begin{tabular}{lccc}
\hline \multicolumn{1}{c}{ Espécies } & $\begin{array}{c}\text { Triportheus } \\
\text { albus } \\
\text { (sardinha } \\
\text { comum) }\end{array}$ & $\begin{array}{c}\text { Triportheus } \\
\text { angulatus } \\
\text { (sardinha } \\
\text { papuda) }\end{array}$ & $\begin{array}{c}\text { Triportheus } \\
\text { auritus } \\
\text { (sardinha } \\
\text { comprida) }\end{array}$ \\
\hline N (espécimes) & 693 & 665 & 486 \\
Lmin. (cm) & 8,4 & 7 & 6,5 \\
Lmáx. (cm) & 23,5 & 25,5 & 26,5 \\
k (por ano) & 0,86 & 0,77 & 0,65 \\
L (cm) & 24,68 & 26,78 & 27,83 \\
$\varnothing^{\prime}$ & 2,71 & 2,74 & 2,70 \\
A 0,95 (ano) & 3,48 & 3,89 & 4,68 \\
${ }^{1}$ M (por ano) & 1,76 & 1,60 & 1,40 \\
${ }^{2}$ M (por ano) & 0,85 & 0,76 & 0,63 \\
a & 0,0398 & 0,0294 & 0,0542 \\
b & 2,6303 & 2,8599 & 2,5094 \\
\hline
\end{tabular}

${ }^{1}$ Pauly (1980); ${ }^{2}$ Taylor (1958)
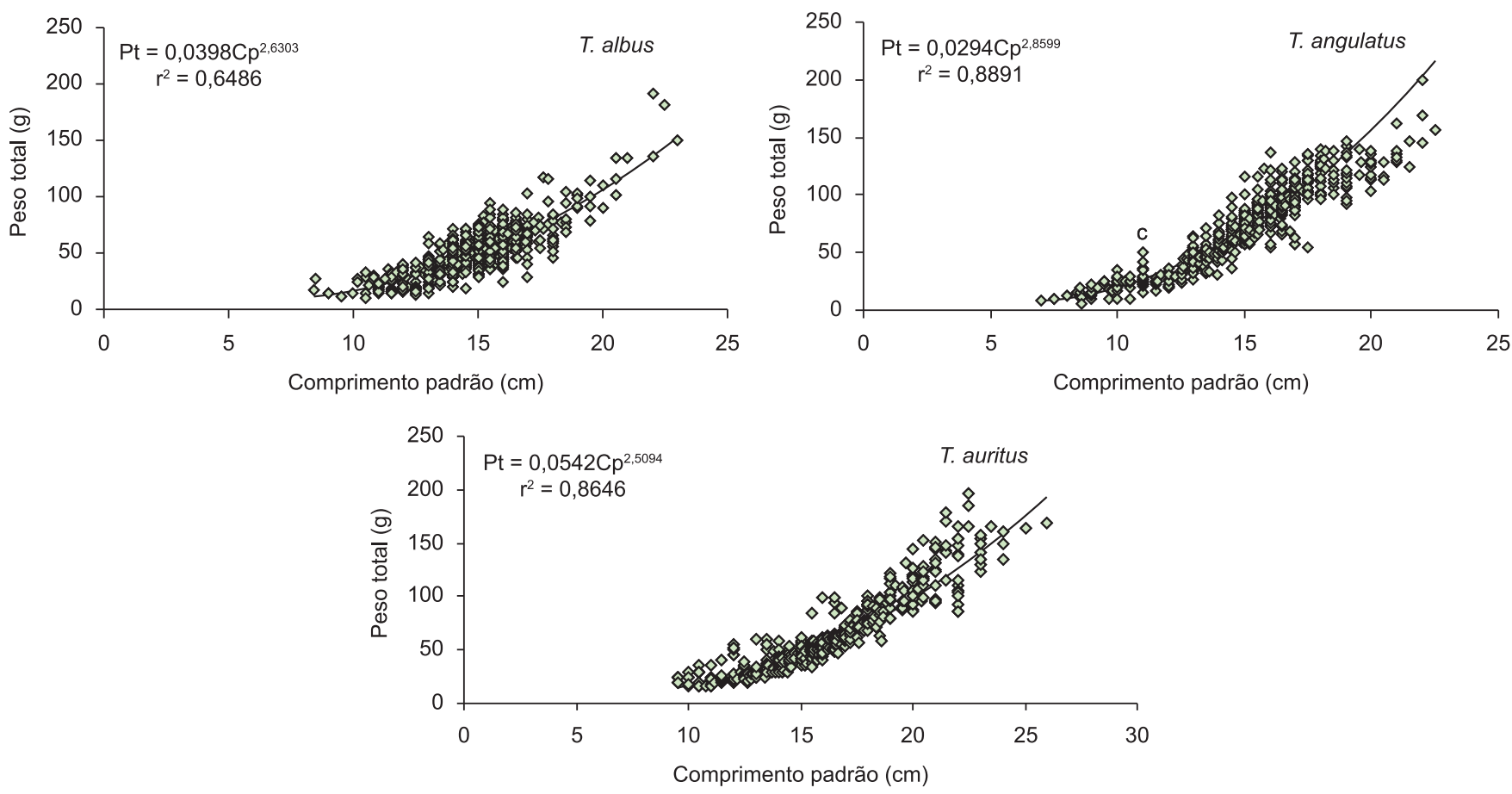

Figura 2. Diagrama de dispersão dos dados de comprimento padrão e peso total de T. albus, T. angulatus e T. auritus capturadas nos lagos Jaitêua e São Lourenço, no Complexo lago Grande de Manacapuru, Rio Solimões, AM.

Figure 2. Scatterplot of standard lenght and total weight of T. albus, T. angulatus and T. auritus captured in Jaitêua and São Lourenço lakes, at the lago Grande de Manacapuru lacustrine complex, Solimões River, AM. 
Tabela 2. Comprimento padrão (cm) de T. albus, T. angulatus e T. auritus nos lagos de várzea da Amazônia Central. Dados: Jaitêua e São Lourenço presente estudo. Rubiano (1999) do lago do Rei. Almeida (1984) do lago do Castanho. Yamamoto et al. (2004) do lago Camaleão.

Table 2. Standard lenght $(\mathrm{cm})$ of T. albus, T. angulatus and T. auritus of studies from flodplains lakes in Central Amazonian. Data: Jaitêua and São Lourenço present study. Rubiano (1999) from Rei lake. Almeida (1984) from Castanho lake. Yamamoto et al. (2004) from Camaleão lake.

\begin{tabular}{lcccc}
\hline \multirow{2}{*}{ Espécies } & \multicolumn{4}{c}{ Classes de comprimento } \\
\cline { 2 - 5 } & Lago Jaiteua e São Lourenço $(\mathbf{c m})$ & Lago Rei $(\mathbf{c m})$ & Lago Castanho & Lago Camaleão \\
\hline Triportheus albus & $8,5-23,5$ & $9,0-19,0$ & - & - \\
Triportheus angulatus & $7,0-25,5$ & $7,0-19,0$ & $9,0-17,0 \mathrm{~cm}$ & $3,0-25,0 \mathrm{~cm}$ \\
Triportheus auritus & $6,5-26,5$ & $10,0-24,0$ & $9,0-21,5 \mathrm{~cm}$ & \\
\hline
\end{tabular}

A taxa de crescimento em peixes é determinada geneticamente e/ou fisiologicamente, enquanto o $\mathrm{L}_{\infty}$ é influenciado pelo suprimento alimentar e densidade populacional (Beverton \& Holt 1957, Sparre \& Venema 1997). Entretanto, há uma interação matemática entre os dois parâmetros, isto é, o valor de $L \infty$ influencia o valor de $k$, e viceversa, condicionando uma variação de $k$ dependente da variação de

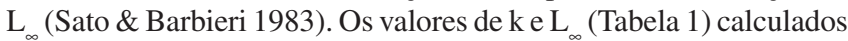
para Triportheus spp. seguiram o padrão acima descrito, sendo que os menores valores de $L \infty$ corresponderam a maiores valores de $k$.

Os parâmetros $\mathrm{k}$ e $\mathrm{L}_{\infty}$ combinados indicam um crescimento rápido, sendo característico de peixes de médio porte que habitam as planícies alagáveis da região Amazônica (Bayley 1988, Amadio \& Villacorta-Correa 1991). Resultados semelhantes foram descritos para Prochilodus nigricans (curimatã) (Ruffino \& Isaac 1995) e Schizodon fasciatus (aracu) (Ruffino \& Isaac 1995). Ambas são espécies de médio porte e r-estrategistas, similar a Triportheus spp. Para complementar as informações de crescimento, foi estimado o índice de performance de crescimento $\left(\emptyset^{\prime}\right)$ (Tabela 1) que busca identificar possíveis diferenças entre estoques em relação às características energéticas, nicho ecológico e erros de estimativas para cada espécie.

Os valores de mortalidade natural foram diferentes conforme o método: Taylor ou Pauly. A estimativa da mortalidade natural (M) conforme método de Pauly (1980) descreve $M$ como sendo uma função da taxa de crescimento (k), comprimento assintótico $\left(\mathrm{L}_{\infty}\right)$ e temperatura do ambiente (T). Considerando que a maioria dos processos biológicos se acelera em condições de altas temperaturas, os altos valores de mortalidade natural provavelmente estão relacionados com altos valores de temperatura ambiental (Sparre \& Venema 1997). Por outro lado, o método de Taylor (1958) relaciona a mortalidade natural (M) com a longevidade (A0,95), que é definida com a idade na qual $95 \%$ da coorte estaria morta se fosse exposta apenas à mortalidade natural. Apesar das diferenças dos valores de acordo com os métodos, as estimativas de mortalidade para as sardinhas seguiram o mesmo padrão: quanto menor o $\mathrm{L}_{\infty}$ maior será o valor de mortalidade natural, independente do método.

A relação peso e comprimento para as três espécies apontou crescimento alométrico negativo. Esse resultado indica que o incremento em comprimento é mais rápido que o incremento em peso, esperado para espécies com formato do corpo alongado e deprimido lateralmente.

Finalmente, é importante mencionar que nos lagos Jaitêua e São Lourenço a heterogeneidade de habitats (Soares et al. 2009) promove condições que favorecem a presença de exemplares de várias classes de tamanho. E, os parâmetros populacionais indicam que T. albus, T. angulatus e T. auritus têm ciclo de vida curto, mortalidade elevada e baixa expectativa de vida (Tabela 1), podendo ser enquadradas na categoria de espécies sazonais (Winemiller 1989). Estes parâmetros, considerados como simples, podem também nos mostrar o estado de "saúde" das espécies. Assim, com a crescente participação das sardinhas no desembarque pesqueiro em Manacapuru e Manaus, esses parâmetros devem ser monitorados para serem utilizados como instrumentos na análise dos estoques pesqueiros.

\section{Agradecimentos}

Agradecemos o apoio financeiro recebido do $\mathrm{MCT} / \mathrm{CNPQ} /$ PPG7, FINEP/CTPetro e Projeto PIATAM. Os autores agradecem aos técnicos, Valter do Santo Dias, João de Sousa Pena e Francisco da Fonseca (INPA) pela substancial ajuda nos trabalhos de campo que foram possíveis somente por causa das suas inestimáveis experiências e dedicações. Somos gratos ao Dr. Ronaldo Angelini pelos valiosas contribuições ao manuscrito.

\section{Referências Bibliográficas}

ALMEIDA, R. G. 1984. Biologia alimentar de três espécies de Triportheus (Pisces: Characoidei: Characidae) do lago Castanho, Amazonas. Acta Amazon. 14(1):48-76.

AMADIO, S.A. \& VILLACORTA-CORREA, M.A. 1991. O estudo de peixes como subsídios ao manejo do recurso pesqueiro. In Bases Científicas para Estratégias de Desenvolvimento da Amazônia; Fatos e Perspectivas (A.L. Val, R. Figliuolo \& E. Feldberg, Ed.). Editora INPA, Manaus, p.333-336.

ANGELINI, R. \& GOMES, L.C. 2008. O artesão de ecossistemas: construindo modelos com dados. Editora Eduem, Maringá.

BARTHEM, R.B. \& PETRERE Jr., M. 1995. Fisheries and populations dynamics of the freshwater catfish Brachyplatystoma vaillantii in the Amazon estuary. In Condition of the World's Aquatic Habitat (N.B. Armantrout, Ed.). Proceedings of the World Fisheries Congress: theme 1. Oxford \& IBH Publishing, New Delhi, p.329-350.

BATISTA, V.S. \& PETRERE Jr., M. 2003. Characterization of the commercial fish production landed at Manaus, Amazonas State, Brazil. Acta Amazon. 33(1):53-66.

BATISTA, V.S. 1998. Distribuição, dinâmica da frota e dos recursos pesqueiros da Amazônia Central. Tese de Doutorado, Instituto Nacional de Pesquisas da Amazônia, Universidade Federal do Amazonas, Manaus.

BAYLEY, P.B. 1988. Factors affecting growth rates of young tropical floodplain fishes: seasonality and density-dependence. Environ. Biol. Fishes 21(2):127-142.

BEVERTON, R.J.H. \& HOLT, S.J. 1957. On the dynamics of exploited fish populations. Fish. Invest. 2(19):1-533.

CUTRIM, L. \& BATISTA, V.S. 2005. Determinação de idade e crescimento do mapará (Hypophthalmus marginatus) na Amazônia Central. Acta Amazon. 35:85-92.

FABRÉ, N.N. \& SAINT-PAUL, U. 1998. Annulus formation on scales and seasonal growth of the Central Amazonian anostomid Schizodon fasciatus. J. Fish. Biol. 53:1-11.

GAYANILO Jr., F.C., SPARRE, P. \& PAULY, D. 1996. The FAO-ICLARM Stock Assessment Tools (FISAT) User's guide. FAO, Roma, 186p. Computerized Information Series Fisheries.

GONÇALVES, C. \& BATISTA, V.S. 2008. Avaliação do desembarque pesqueiro efetuado em Manacapuru, Amazonas, Brasil. Acta Amazon. 38(1):135-144.

IBAMA 2008. Estatística da Pesca 2006 Brasil: grandes regiões e unidades da federação. IBAMA, Brasília. 
ISAAC, V.J. \& RUFFINO, M.L. 1996. Population dynamics of tambaqui, Colossoma macropomum Cuvier, in the Lower Amazon, Brazil. Fish. Manag. Ecol. 3:315-333.

MERONA, B. \& BITTENCOURT, M.M. 1988. A pesca na Amazônia através dos desembarques no smercado de Manaus: resultados preliminares. Mem. Soc. Cienc. Nat. La Salle 48:433-453.

MOREAU, J. 1987. Mathematical and biological expression of growth in fishes: recent trends and further developments. In The age and growth of fish (R.C. Summerfelt \& G.E. Hall, Ed.). Iowa State University Press, Iowa, p.81-113.

PAULY, D. \& MUNRO, J.L. 1984. Once more on the comparison of growth in fish and invertebrates. ICLARM Fishbyte Newsletter of the Network of Tropical Fisheries Scientists 2(1):72-94.

PAULY, D. 1980. On the interrelationships between natural mortality, growth parameters and mean environmental temperature in 175 fish stocks. J. Cons. Int. Explor. Mer. 39(3):175-192.

PAULY, D. 1994. Quantitative analysis of published data on the growth, metabolism, food consumption, and related features of the red-bellied piranha, Serrasalmus nattereri (Characidae). Environ. Biol. Fishes 41:423-437.

PENNA, M.A.H., VILLACORTA-CORRÊA, M.A., WALTER, T. \& PETRERE Jr., M. 2005. Growth of the Tambaqui Colossoma macropomum (Cuvier) (Characiformes: Characidae): Which is the best model? Braz. J. Biol. 65(1):129-139.

PEREZ, A. \& FABRÉ, N.N. 2009. Seasonal growth and life history of the cat fish Calophysus macropterus (Lichtenstein, 1819) (Siluriformes: Pimelodidae) from the Amazon floodplain. J. Appl. Ichthyol. 25:343-349.

PETRERE Jr., M. 1978. Pesca e esforço de pesca no estado do Amazonas. II - Locais e aparelhos de captura e estatística de desembarque. Acta Amazon. 8(2):1-54.

PETRERE Jr., M. 1983. Yield per recruit of the Tambaqui, Colossoma macropomum Cuvier, in the Amazon State, Brazil. J. Fish. Biol. 22:133-144.

PETRERE Jr., M. 1992. As comunidades humanas ribeirinhas da Amazônia e suas transformações sociais. In Populações humanas, rios e mares da Amazônia São Paulo (A.C. Diegues, Ed.). Anais do IV Encontro de Ciências Sociais e o Mar no Brasil. São Paulo, p.31-68.
RUBIANO, A. L. M. 1999. Táticas reprodutivas de espécies de Characiformes em área de várzea na Amazônia Central (Lago do Rei). Dissertação de Mestrado, Instituto Nacional de Pesquisas do Amazonas, Universidade do Amazonas, Manaus.

RUFFINO, M.L. \& ISAAC, V.J. 1995. Life cycle and Biological Parameteres of Several Brazilian Amazon Fish Species. NAGA: The ICLARM Quarterly 18(4):41-45.

RUFFINO, M.L., SOARES, E.C., SILVA, C.O., BARTHEM, R.B., BATISTA, V., ESTUPIÑÁN, G. \& PINTO, W. 2006. Estatística Pesqueira do Amazonas e Pará, 2003. IBAMA, ProVárzea, Manaus.

SATO, Y. \& BARBIERI, G. 1983. Crescimento de Schizodon Knerii Steindachner, 1875 (Pisces, Anostomidae) na represa de Três Marias, Minas Gerais. An. Sem. Reg. Ecol. 3:201-221.

SMITH, N.J. 1979. A pesca no Rio Amazonas. Editora INPA, Manaus.

SOARES, M.G.M., SILVA, F.R., ANJOS, H.D.B., PRESTES, L., BEVILAQUA, D.R. \& CAMPOS, C.P. 2009. Ambientes de pesca e a ictiofauna do complexo lacustre do lago grande de Manacapuru, AM: Composição taxonômica e Parâmetros populacionais. In A pesca na Amazônia Central - Ecologia, conhecimento tradicional e formas de manejo (T.J.P. Fraxe \& A.C. Witkoski Eds.). Editora EDUA, Manaus, p.59-89.

SPARRE, P. \& VENEMA, S.C. 1997. Introdução à avaliação de mananciais de peixes tropicais. Parte 1: Manual. FAO, Roma.

TAYLOR, C.C. 1958. Cod growth and temperature. J. Cons. Int. Explor. Mer. 23:366-370.

WERDER, U. 1983. Age determination by scale analysis in juvenile matrinchã (Brycon cf. melanopterus) (Muller and Troscheli Teleostei: Characoidei) a tropical characin from the Central Amazon. Amazoniana 8:445-464.

WINEMILLER, K.O. 1989. Patterns of variation in life history among South American fishes in seasonal environments. Oecologia 81:225-241.

YAMAMOTO, K.C., SOARES, M.G.M. \& FREITAS, C.E.C. 2004 Alimentação de Triportheus angulatus (Spix and Agassiz, 1829) no lago Camaleão, Manaus, AM, Brasil. Acta Amazon. 34(4):653-659.

ZAR, J.H. 1996. Biostatistical Analysis. Prentice-Hall, London, England.

Recebido em 26/07/2010

Versão reformulada recebida em 20/08/2010

Publicado em 25/08/2010 